\title{
Alzheimer's Disease and Retinal Neurodegeneration Share a Consistent Stress Response of the Neurovascular Unit
}

\author{
Stephanie Busch ${ }^{a}$ Liang Wu ${ }^{a}$ Yuxi Feng ${ }^{a, b} \quad$ Norbert Gretz ${ }^{c}$ Sigrid Hoffmann ${ }^{c}$ \\ Hans-Peter Hammes ${ }^{\text {a }}$ \\ a5th Medical Department, 'DInstitute of Experimental and Clinical Pharmacology and Toxicology, \\ and 'Medical Research Center, Medical Faculty Mannheim, University of Heidelberg, Germany
}

\section{Key Words}

Alzheimer's disease - Retinopathy - Neurodegeneration - Neurovascular unit - Polycystic kidney disease

\begin{abstract}
Background: The pathogenesis of Alzheimer's disease (AD) is characterized by neuronal injury, activation of microglia and astrocytes, deposition of amyloid- $\beta$ and secondary vessel degeneration. In the polycystic kidney disease (PKD) rat model, we observed neuronal injury, microglial activation and vasoregression. We speculated that this neuroretinal degeneration shares important pathogenetic steps with AD. Therefore, we determined the activation of astrocytes and the accumulation of amyloid- $\beta$ in PKD retinae. Methods: Immunohistochemistry of PKD retinae for vimentin, carboxymethyllysin, beta-Amyloid 1-42, High-Mobility-GroupProtein B1 and amyloid protein precursor was performed. Results: Adjunct to astrocyte activation, accumulation of beta-Amyloid 1-42 and High-Mobility-Group-Protein B1 in astrocytes and around vessels of the superficial network was found in PKD retinae prior to the onset of vasoregression. Amyloid precursor protein was localized adjacent to the outer segment of photoreceptors in PKD and control rats. The parallel appearance of AD-related peptides indicates an alarmine based response to photoreceptor degeneration and secondary vasoregression. Conclusion: The model has broad overlap with $A D$ and may be suitable to study beneficial pharmacological concepts.
\end{abstract}

Copyright $(2012$ S. Karger AG, Basel

\section{Introduction}

Alzheimer's disease (AD) is characterized by chronic and progressive neurodegeneration and accumulation of neurotoxic amyloid- $\beta$ and neuronal injury [1]. However, the neurovascular unit is also important in the pathogenesis of $\mathrm{AD}$ [2-3]. Activation of the immune system in $\mathrm{AD}$ 
is reflected by enhanced microglial activation, upregulation of the complement system and an increased release of chemokines and cytokines [4]. As a marker for microglial activation, CD74, the invariant chain of MHC II, is upregulated in AD [5]. End stage AD leads to vascular dysfunction through degeneration of endothelial cells and pericytes, accumulation of amyloid- $\beta$ in vessel walls and formation of acellular capillaries [6]. Zlokovic et al. classified four phases in AD's pathogenesis at the blood brain barrier: 1 . early phase with neuronal injury by neurotoxic amyloid- $\beta, 2$. early symptomatic phase characterized by activation of endothelial cell, pericytes, microglia and astrocytes, 3. late symptomatic phase shown by deposition of amyloid- $\beta$ on vessel walls and degeneration of endothelial cells and pericytes and 4. end stage defined through collaps of the capillaries [3].

The transgenic polycystic kidney disease (PKD) rat is a model of ciliopathy [7]. The PKD rat displays retinal photoreceptor degeneration starting at one month of age in parallel to glial activation shown by upregulation of glial fibrillary acidic protein (GFAP). At the second month of age the numbers of endothelial cells and pericytes decrease and acellular capillaries start to form exponentially [8]. Gene analysis revealed upregulation of components of the innate immune system following neuronal injury but preceded vascular regression [9].

Thus, $\mathrm{AD}$ and the retinal phenotype of the PKD rat share neurodegeneration (by neurotoxic amyloid- $\beta$ or genetically driven), activation of microglia, shown by upregulation of CD74, and finally vasoregression. Other factors affecting the pathogenesis of AD like activation of astrocytes and accumulation of amyloid- $\beta$ by cleavage of amyloid protein precursor (APP) have not been determined in the PKD rat.

The purpose of this study was to establish the PKD model as a retinal correspondent to AD. Immunohistochemistry of PKD retinae for vimentin, as a marker of astrocyte activation, and AD-related proteins carboxymethyllysin (CML), beta-Amyloid 1-42 and High-MobilityGroup-Protein B1 (HMGB1) were performed. To further evaluate the origin of beta-Amyloid deposition, APP staining occurred.

\section{Materials and Methods}

\section{Animals}

Generation and genotyping of the PKD-2-247 (PKD) rats expressing a truncated human polycystic-2 gene has been described previously [7]. The rats were held in a 12 hours light and dark cycle with free access to food and drinking water. In this study heter- and homozygote PKD rats were used. Spraque-Dawley (SD) rats were held as controls. At 1, 2 and 3 months the rats were anesthetized and sacrificed. The eyes were enucleated and immediately frozen at $-80^{\circ} \mathrm{C}$ or fixed in $4 \%$ formalin.

This study was admitted by the ethic committee Regierungspräsidium Karlsruhe, approval ID: 359185.81/G-219/10.

\section{Immunohistochemistry of vimentin}

The eyes of 2 months old PKD and SD rats were immediately frozen at $-80^{\circ} \mathrm{C}$. After embedding in tissue-tek the eyes were sectioned at $-22^{\circ} \mathrm{C}$ to $6 \mu \mathrm{m}$. They were dried at room temperature for $24 \mathrm{~h}$ and then stored at $-80^{\circ} \mathrm{C}$ until staining. After thawing for $30 \mathrm{~min}$ at room temperature the tissue was fixed with $-20^{\circ} \mathrm{C}$ acetone for $10 \mathrm{~min}$. Incubation with blocking solution containing $1 \%$ bovine serum albumin (BSA) in phosphate-buffered saline solution (PBS) for 30min prevented unspecific protein interaction. Primary antibody rabbit anti-rat vimentin (1:300; abcam, Cambridge, United Kingdom) was diluted in 1\% BSA and $0.5 \%$ triton $\mathrm{x}-100$ in PBS and incubated at room temperature for $1 \mathrm{~h}$. Slices were washed three times with PBS. Incubation with the secondary antibody swine anti-rabbit labeled with FITC (1:300; Dako, Hamburg, Germany) diluted as described before followed for 1h. After washing,4',6-diamidino-2-phenylindole (DAPI; Sigma, Munich, Germany) was used for nuclei staining. Final washing steps followed and slices were covered with 50\% glycerol. Photographs were taken with an Olympus BX51 microscope (Olympus Opticals Europe, Hamburg, Germany). 
Immunohistochemistry of amyloid precursor protein

Eyes of 3 months old PKD and SD rats were cut into sections and fixed as described before. They were blocked with $5 \%$ donkey serum in $1 \%$ BSA solution for 30min. Primary antibody rabbit anti-rat amyloid precursor protein (1:100; abcam, Cambridge, United Kingdom) was diluted in 1\% BSA and incubated at room temperature for $1 \mathrm{~h}$. Slices were washed three times with PBS. Incubation with the secondary antibody donkey anti-rabbit DyLight 488 (1:100; Jackson ImmunsResearch, Pennsylvania, United States) diluted in PBS with $1 \%$ normal rat serum occurred for $30 \mathrm{~min}$. After washing, sections were covered with Vectashield mounting medium with DAPI (Vector laboratories, Burlingame, United States). Photographs were taken with a Leica DM4000B microscope (Leica, Wetzlar, Germany).

\section{Immunohistochemistry of paraffinsections}

The eyes of 1 and 3 months old PKD and SD rats were embedded into paraffin and cut to a thickness of $6 \mu \mathrm{m}$. After dewaxing and rehydrating the sections were microwaved in citrate buffer for $20 \mathrm{~min}$. Sections were incubated with $0.5 \%$ triton $\mathrm{x}-100$ for permeabilization. Unspecific protein interaction was avoided by incubation with $1 \%$ BSAfor $30 \mathrm{~min}$ at room temperature. After blocking sections were incubated with their appropriate primary antibody diluted in PBS overnight at $4^{\circ} \mathrm{C}$ : mouse anti-rat carboxymethyllysin (CML-2F8, 1:5000; Novo Nordisk, Bagsvaerd, Denmark), rabbit anti-rat High-Mobility-Group-Protein B1 (HMGB1, 1:1000; Millipore, Billerica, United States) or rabbit anti-rat beta-Amyloid 1-42 (1:100; abcam, Cambridge, United Kingdom). After washing three times in PBS, incubation with the secondary FITC-labeled antibody rabbit anti-mouse or swine anti-rabbit (1:20; Dako, Hamburg, Germany) diluted in PBS for $1 \mathrm{~h}$ at room temperature followed. Sections were washed with PBS three times and mounted in 50\% glycerol. A confocal microscope (Leica TCS SP2 Confocal Microscope; Leica, Wetzlar, Germany) was used to take the photographs.

\section{Results}

Glial activation in PKD retinae

To investigate glial activation staining for vimentin, a mesenchymal intermediate filament which is upregulated during gliosis, occurred (Fig. 1). Vimentin expression in 2 months Spraque-Dawley (SD) retinae was limited to the ganglion cell layer and the inner plexiform layer (Fig. 1 A, B). The morphology of these cells was characteristic for astrocytes. In PKD retinae of the same age immunolabeling was substantially stronger and labeled predominantly soma of astrocytes (Fig. 1, arrow) localized in the ganglion cell layer (Fig. $1 \mathrm{C}$, D). The staining of the filamentous bundles (Fig. 1, arrowhead) appeared stronger and more extended into the outer part of the retina than in SD rats. Thus, vimentin staining illustrated activation of astrocytes in the PKD rat.

\section{Accumulation of AD-related proteins in PKD retinae}

Three different immunohistological stainings for the AD-related proteins CML, HMGB1 and amyloid- $\beta$ were used to investigate the possible overlap phenotypes between $A D$ and PKD rat. We found a stronger expression of all proteins in PKD than in SD rats, predominantly located in the ganglion cell layer, the inner plexiform layer and surrounding vessels of the superficial network (Fig. 2). Carboxymethyllysin (CML), an advanced glycation end product which accumulates in neurons and glial cells during aging and in AD, was not detectable in SD retinae of either 1 or 3 months (Fig. 2 A, D) [10]. In PKD retinae CML-labeling was weakly positive in the ganglion cell layer and in cells surrounding vessels of the superficial network (Fig. 2 G arrow, J).

High-mobility group protein 1 (HMGB1) is expressed by apoptotic neurons and upregulated in $\mathrm{AD}$ [11-12]. In SD retinae we found HMGB1-labeling of ganglion cells in an age-dependent manner (Fig. 2 E arrowhead). The expression in PKD retinae was confined to the inner retinal part. Their typical morphology of transversal filamentous bundles identified the cells as glia (Fig. $2 \mathrm{H}$ star). HMGB1 was also found surrounding superficial vessels (Fig. $2 \mathrm{~K}$ arrow). 
Fig. 1. Expression of vimentin in vertical sections of 2 months $\mathrm{SD}(\mathrm{A}-\mathrm{B})$ and $\mathrm{PKD}(\mathrm{C}-\mathrm{D})$ retinae. 200x (A, C) and 400x (B, D) original magnification. Vimentin is stained in green, cell nuclei are displayed by 4,6-diamidino2-phenylindole (DAPI). An increase of vimentin expression in PKD retinae, predominantly in astrocyte's soma (arrow) and filamentous bundles (arrowhead) is observable. GCL ganglion cell layer, IPL inner plexiforme layer, INL inner nuclear layer, ONL outer nuclear layer.

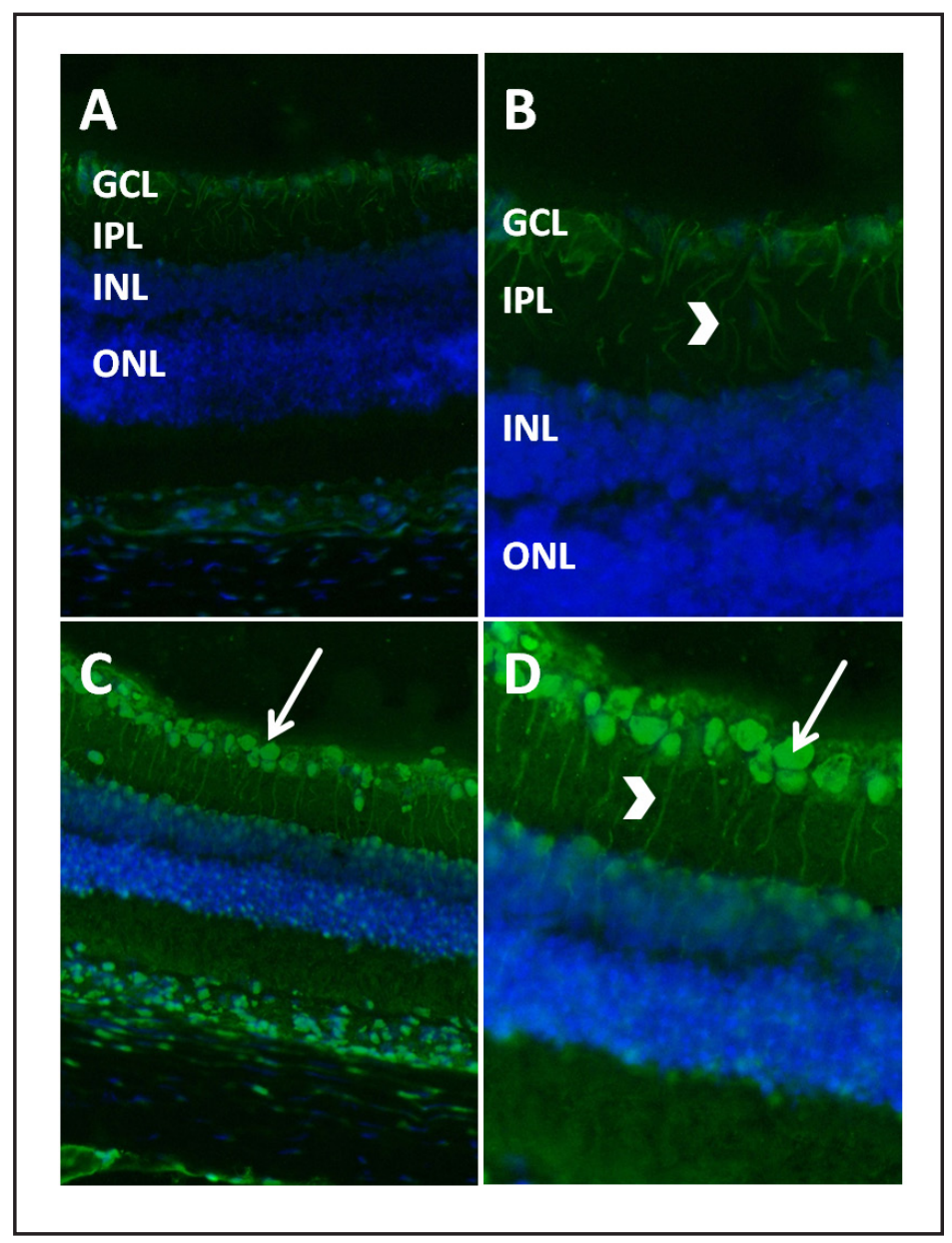

The staining for beta-Amyloid 1-42 ( $\beta$ A1-42), a protein which typically accumulates in $A D$, revealed the explicit difference between SD and PKD rats. SD retinae showed weak staining surrounding superficial (Fig. $2 \mathrm{C}$ arrow) vessel and no expression of $\beta$ A1-42. In PKD retinae immunolabeling was strongly positive at the inner retinal part and surrounding superficial vessels (Fig. 2 I, L). Therefore, the neurodegenerative PKD shares the accumulation of AD-related proteins with $\mathrm{AD}$.

\section{APP expression in the outer segment is not altered in the PKD model}

Given that beta-Amyloid is one of the cleavage products of APP, staining for APP occurred to test if it is also altered in the PKD model (Fig. 3). The expression of APP in retinae of 3 months SD (Fig. 3, A and B) and PKD (Fig. 3, C and D) rats was exclusively in outer segment of photoreceptors. There was no difference in expression of APP in SD and PKD retinae. The staining pattern is not characteristic for any cell type localized in this part of the retina, which suppose an extracellular localization of APP.

\section{Discussion}

This study reveals that the PKD model phenocopies important neurovascular patterns of $\mathrm{AD}$ in the retina, suggesting a uniform stress response in the neurovascular unit of brain and retina.

Vimentin staining in 2 months old PKD rats indicated astrocyte activation [13]. This observation completes the previously shown GFAP upregulation as sign of glial activation 
Cellular Physiology and Biochemistry
Cell Physiol Biochem 2012;30:1436-1443

\begin{tabular}{|l|l|}
\hline DOI: $10.1159 / 000343331$ & C 2012 S. Karger AG, Basel
\end{tabular}

www.karger.com/cpb

1440

Fig. 2. Expression of Alzheimer's Disease related proteins in SD $(\mathrm{A}-\mathrm{F})$ and $\mathrm{PKD}(\mathrm{G}-\mathrm{L})$ retinae at 1 (A-C, G-I) and 3 (D-F, J-L) months. Carboxymethyllysin (CML), Highmobility group protein 1 (HMGB1) and beta-Amyloid 1-42 (ßA1-42) are stained in green and reveal a stronger expression in PKD retinae. Arrows point at positive staining around vessels of the superficial network. Arrowhead faces towards ganglion cells. The star shows transversal filamentous bundles. GCL ganglion cell layer, INL inner nuclear layer.

Busch/Wu/Feng/Gretz/Hoffmann/Hammes: Neurovascular Unit in PKD and AD

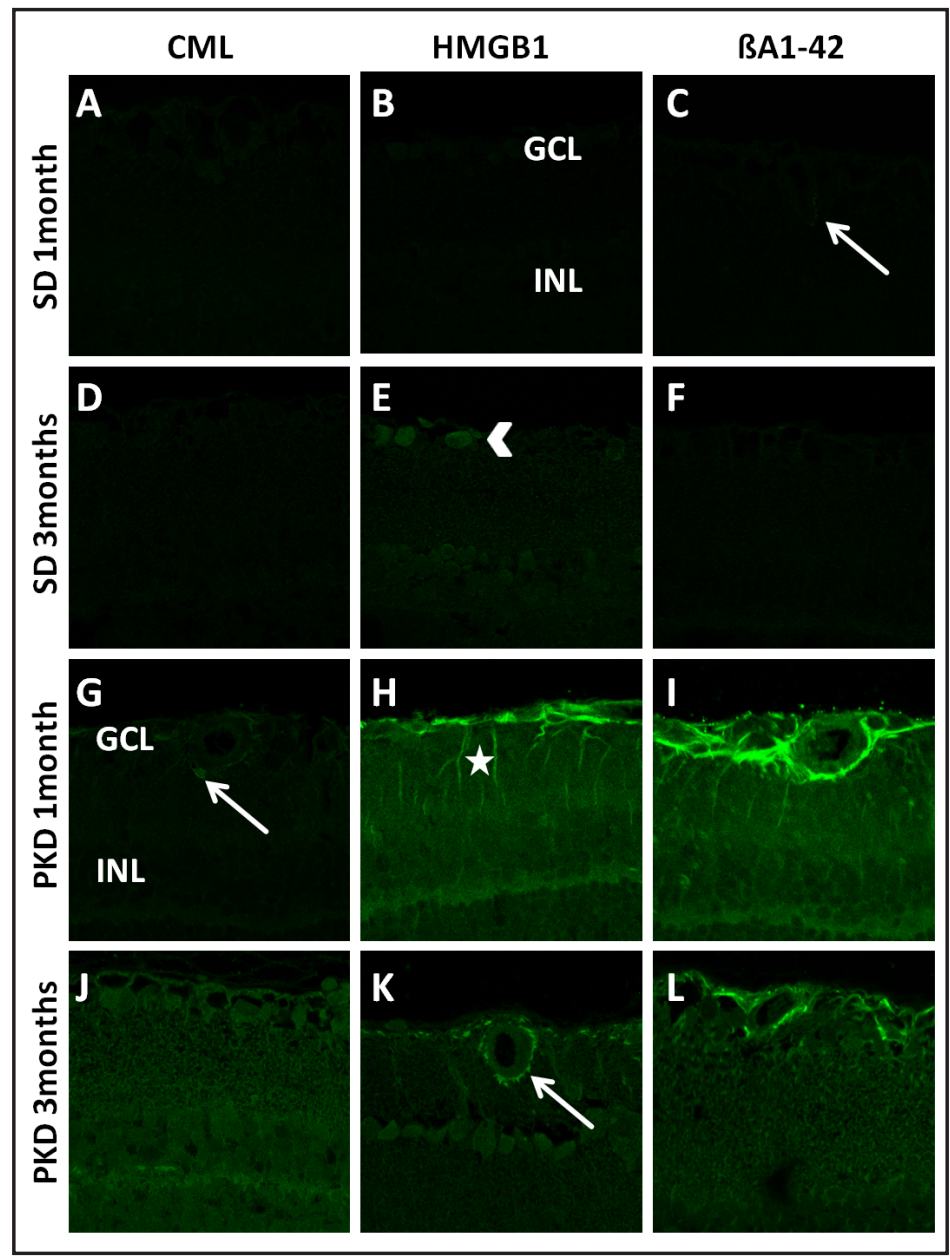

Fig. 3. Expression of amyloid protein precursor (APP) in vertical sections of 3 months SD (A-B) and PKD (C-D) retinae. 200x (A, C) and 400x (B, D) original magnification. APP is stained in green, cell nuclei are displayed by 4',6-diamidino2-phenylindole (DAPI). APP is intraretinal exclusively expressed in the outer segment of the photoreceptor. There is no change in staining between SD and PKD rats. GCL ganglion cell layer, INL inner nuclear layer, ONL outer nuclear layer, OS outer segment.

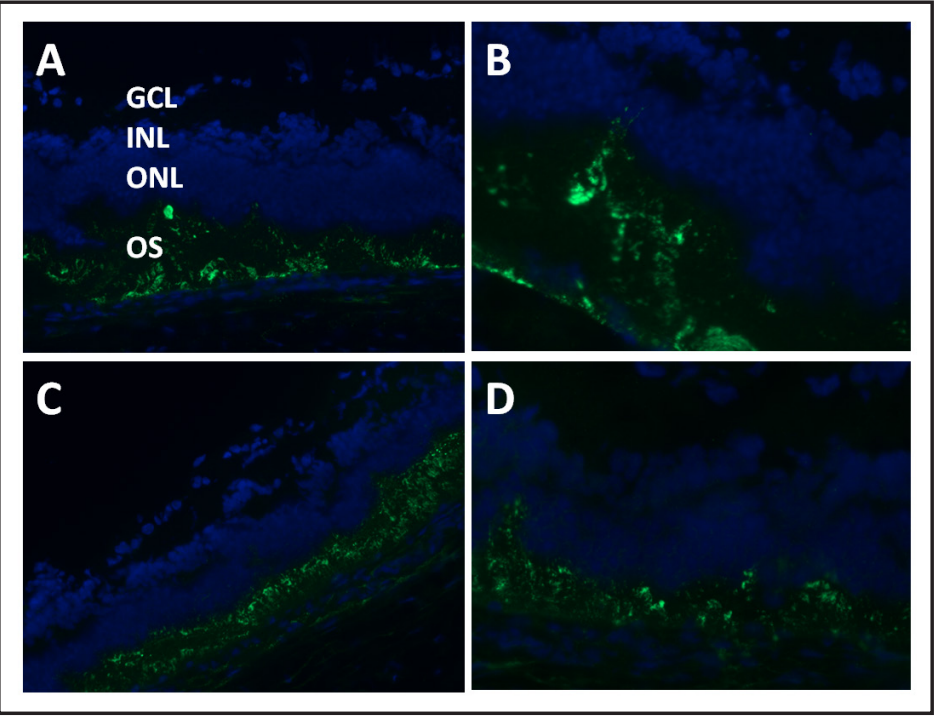

in the PKD model [8]. Higher expression of vimentin is attributed to an increase of basic fibroblast growth factor (bFGF), which is fourfold elevated in 1 month old PKD rats, and is reported to be a stimulus for vimentin upregulation $[8,14]$. 
Parallel to astrocyte activation our experiments display upregulation of amyloid- $\beta$ and HMGB1 in the ganglion cell layer (GCL), predominantly in a typical glial pattern and around vessels of the superficial vessel network. Amyloid- $\beta$ colocalized with glutaminsyntheatase, a marker for Müller cells, indicating the expression of amyloid- $\beta$ by Müller cells (data not shown). Similarly, beta-amyloid precursor protein is found in Müller cells of inherited retinal dystrophy which is a form of proliferative vitreoretinopathy [15-16]. HMGB1 is a damageassociated molecular pattern which is amongst others expressed by degenerating neurons and functions as a inflammatory cytokine when released to the extracellular matrix [17]. HMGB1 is elevated in AD and it colocalizes with amyloid- $\beta$ suggesting that it influences the homeostasis of amyloid- $\beta$ [18]. Furthermore, HMGB1 can activate microglia through the receptor for advanced glycation end products which is upregulated in $\mathrm{AD}$ and macrophage antigen complex 1 [19-20]. In the PKD rat, HMGB1 staining is positive in the ganglion cell layer and around vessels of the superficial layer. 3 months old control SD rats also show slight staining for HMGB1 in a typical ganglion cell pattern. This could be an age-dependent effect. HMGB1 also colocalized with the Müller cell marker glutaminsynthetase (data not shown).

In contrast to the strong positive staining of amyloid- $\beta$ and HMGB1, CML showed an unanticipated moderate staining around superficial vessels. In AD the advanced glycation end product (AGE) CML is upregulated in neurons and cerebral vessels [21]. CML upregulation has been shown in other retinal diseases like diabetic retinopathy and age-related macular degeneration [22-24]. The contrasting results are attributable to the lack of hyperglycemia in the PKD model, which is required for formation of AGEs.

Due to the fact that beta-Amyloid is a cleavage product of APP, a staining for APP was performed [25]. Interestingly APP was exclusively expressed in the area of the outer segment of photoreceptors and its expression was not altered in the PKD model. This suggests a change in secretase expression from $\alpha$-secretase, producing the neuroprotective soluble peptide $\operatorname{APP} \alpha$, to $\beta$ - and $\gamma$-secretase, producing the accumulating and potential neurotoxic beta-Amyloid [26].

\section{Conclusion}

The retinal neurodegenerative PKD model is a phenocopy of AD. Both share the chronology of neurodegeneration, activation of astrocytes and microglia, accumulation of AD-related proteins and subsequent vasoregression. Thus, we assume this pathogenesis not to be specific for a single disease but rather a common stress response of the neurovascular unit and the innate immunie system.

The PKD model has broad overlap with AD. It shares important pathogenic steps with AD like neuronal damage, glial activation, accumulation of beta-Amyloid and vasoregression. Therefore it may be suitable to study beneficial pharmacological concepts.

\section{Abbreviations}

AD (Alzheimer's disease); AGE (advanced glycation end product); APP (amyloid precursor protein); bFGF (basic fibroblast growth factor); $\beta A 1-42$ (beta-Amyloid 1-42); CD74 (invariant chain of MCH II); CML (carboxymethyllysin); GCL (ganglion cell layer); GFAP (glial fibrillary acidic protein); HMGB1(High-Mobility-Group-Protein B1); INL (inner nuclear layer); ONL (outer nuclear layer); OS (outer segment); PKD )polycystic kidney disease).

\section{Competing interests}

The author has no competing interests. 


\begin{tabular}{|c|c|c|}
\hline Cellular Physiology & Cell Physiol Biochem 2012;30:1436-1443 & \\
\hline and Biochemistry & $\begin{array}{l}\text { DOI: 10.1159/000343331 } \\
\text { Published online: November 22, } 2012\end{array}$ & $\begin{array}{l}\text { O } 2012 \text { S. Karger AG, Basel } \\
\text { www.karger.com/cpb }\end{array}$ \\
\hline
\end{tabular}

\section{Acknowledgements}

This work was supported by the Deutsche Forschungsgemeinschaft (GRK 880; S.B.). The author is grateful to P. Bugert, N. Dietrich, V. Faltermann and U. Kaiser for advice and support.

\section{References}

1 Hardy J, Selkoe DJ: The amyloid hypothesis of Alzheimer's disease: progress and problems on the road to therapeutics. Science 2002;297:353-356.

2 Koistinaho M, Kettunen MI, Goldsteins G, Keinanen R, Salminen A: Beta-amyloid precursor protein transgenic mice that harbor diffuse A beta deposits but do not form plaques show increased ischemic vulnerability: role of inflammation. Proc Natl Acad Sci USA 2002;99:1610-1615.

3 Zlokovic BV:The blood-brain barrier in health and chronic neurodegenerative disorders. Neuron 2008;57:178-201.

4 Heneka MT, O'Banion MK: Inflammatory processes in Alzheimer's disease. J Neuroimmunol 2007;184:6991.

5 Demos C, Bandyopadhyay M, Rohrer B: Identification of candidate genes for human retinal degeneration loci using differentially expressed genes from mouse photoreceptor dystrophy models. Mol Vis 2008;14:1639-1649.

6 Farkas E, Luiten PG: Cerebral microvascular pathology in aging and Alzheimer's disease. Prog Neurobiol 2001;64:575-611.

7 Gallagher AR, Hoffmann S, Brown N, Cedzich A, Meruvu S: A truncated polycystin-2 protein causes polycystic kidney disease and retinal degeneration in transgenic rats. J Am Soc Nephrol 2006;17:27192730.

8 Feng Y, Wang Y, Stock O, Pfister F, Tanimoto N: Vasoregression linked to neuronal damage in the rat with defect of polycystin-2. PLoS One 2009;4:e7328.

9 Feng Y, Wang Y, Li L, Wu L, Hoffmann S: Gene expression profiling of vasoregression in the retina-involvement of microglial cells. PLoS One 2011;6:e16865.

10 Takeda A, Wakai M, Niwa H, Dei R, Yamamoto M: Neuronal and glial advanced glycation end product [Nepsilon-(carboxymethyl)lysine]] in Alzheimer's disease brains. Acta Neuropathol 2001;101:27-35.

11 Takata K, Takada T, Ito A, Asai M, Tawa M: Microglial Amyloid-beta1-40 Phagocytosis Dysfunction Is Caused by High-Mobility Group Box Protein-1: Implications for the Pathological Progression of Alzheimer's Disease. Int J Alzheimers Dis 2012:685739.

12 Kawabata H, Setoguchi T, Yone K, Souda M, Yoshida H: High mobility group box 1 is upregulated after spinal cord injury and is associated with neuronal cell apoptosis. Spine (Phila Pa 1976) 2010;35:1109-1115.

13 de Rivero Vaccari JP, Minkiewicz J, Wang X, De Rivero Vaccari JC, German R: Astrogliosis involves activation of retinoic acid-inducible gene-like signaling in the innate immune response after spinal cord injury. Glia 2012;60:414-421.

14 Lewis GP, Erickson PA, Guerin CJ, Anderson DH, Fisher SK: Basic fibroblast growth factor: a potential regulator of proliferation and intermediate filament expression in the retina. J Neurosci 1992;12:39683978.

15 Hartig W, Grosche J, Distler C, Grimm D, el-Hifnawi E: Alterations of Muller (glial) cells in dystrophic retinae of RCS rats. J Neurocytol 1995;24:507-517.

16 McGillem GS, Dacheux RF: Rabbit retinal Muller cells undergo antigenic changes in response to experimentally induced proliferative vitreoretinopathy. Exp Eye Res 1999;68:617-627.

17 Dvoriantchikova G, Hernandez E, Grant J, Santos AR, Yang H: The high-mobility group box-1 nuclear factor mediates retinal injury after ischemia reperfusion. Invest Ophthalmol Vis Sci 2011;52:7187-7194.

18 Takata K, Kitamura Y, Kakimura J, Shibagaki K, Tsuchiya D: Role of high mobility group protein-1 (HMG1) in amyloid-beta homeostasis. Biochem Biophys Res Commun 2003;301:699-703. 
19 Yan SD, Chen X, Fu J, Chen M, Zhu H: RAGE and amyloid-beta peptide neurotoxicity in Alzheimer's disease. Nature 1996;382:685-691.

20 Gao HM, Zhou H, Zhang F, Wilson BC, Kam W: HMGB1 acts on microglia Mac1 to mediate chronic neuroinflammation that drives progressive neurodegeneration. J Neurosci 2011;31:1081-1092.

21 Southern L, Williams J, Esiri MM: Immunohistochemical study of N-epsilon-carboxymethyl lysine (CML) in human brain: relation to vascular dementia. BMC Neurol 2007;7:35.

22 Stitt A, Gardiner TA, Alderson NL, Canning P, Frizzell N: The AGE inhibitor pyridoxamine inhibits development of retinopathy in experimental diabetes. Diabetes 2002;51:2826-2832.

23 Hammes HP, Alt A, Niwa T, Clausen JT, Bretzel RG: Differential accumulation of advanced glycation end products in the course of diabetic retinopathy. Diabetologia 1999;42:728-736.

24 Hammes HP, Hoerauf H, Alt A, Schleicher E, Clausen JT: N(epsilon)(carboxymethyl)lysin and the AGE receptor RAGE colocalize in age-related macular degeneration. Invest Ophthalmol Vis Sci 1999;40:18551859.

25 Nunan J, Small DH: Regulation of APP cleavage by alpha-, beta- and gamma-secretases. FEBS Lett 2000;483:6-10.

26 Chasseigneaux S, Allinquant B: Functions of Abeta, sAPPalpha and sAPPbeta : similarities and differences. J Neurochem 2012;120:S99-108. 